\title{
ANATOMÍA ECONÓMICA DE MARA (DOLICHOTIS PATAGONUM) Y SU APLICACIÓN EN LA EVALUACIÓN DE RESTOS ZOOARQUEOLÓGICOS RECUPERADOS EN LA COSTA NORPATAGÓNICA (RÍO NEGRO, ARGENTINA)
}

HERNÁN A. MARANI

\begin{abstract}
RESUMEN
En este trabajo se presenta el índice de utilidad económica de Dolichotis patagonum (mara). Dicho índice será utilizado como marco de referencia en la evaluación de la evidencia arqueofaunística del consumo temprano de este roedor en la costa Norpatagónica. Se plantea una discusión acerca del potencial de esta especie como recurso alimenticio y se interpretan los recientes descubrimientos arqueológicos en el contexto de la subsitencia de los grupos cazadores-recolectores de la costa norte del golfo San Matías. De esta forma, la aplicación del índice presentado en este trabajo permitirá evaluar la representación de partes esqueletarias registradas en los diferentes sitios arqueológicos de está porción meridional de Sudamérica.

PALABRAS CLAVE: Anatomía económica, Dolichotis patagonum, Norpatagonia, zooarqueología. ECONOMIC ANATOMY OF MARA (DOLICHOTIS PATAGONUM) AND ITS
APPLICATION TO EVALUATE THE ZOOARCHAEOLOGICAL REMAINS
RECOVERED IN NORTHERN PATAGONIA COAST (RÍO NEGRO, ARGENTINA)
\end{abstract}

\section{ABSTRACT}

The following work presents an economic utility index for Dolichotis patagonum (mara). This index can be used as a reference in the evaluation of early consumption of this rodent in archaeofaunal assemblages in the north coast of Patagonia. The potential of this species as a nutritious resource for the hunter-gatherer groups of the north cost of the gulf of San Matías, as well as the recent archaeological findings are discussed. In this context, the application of the index presented in this work permitted the evaluation of the representation of skeletal parts registered in the different archaeological sites in this portion of southern South America.

KEY WORDS: economic anatomy, Dolichotis patagonum, Northern Patagonia, zooarchaeology. 


\section{INTRODUCCIÓN}

Durante las excavaciones realizadas en el año 2008 en el marco de las investigaciones que vienen desarrollándose en la costa norte del golfo San Matías (Favier et al. 2006; Borella et al. 2007; Favier Dubois y Borella 2007; entre otros) se recuperaron gran cantidad de restos óseos asignado a mara (Dolichotis patagonum). La presencia de estos restos en concheros arqueológicos con cronología temprana (principios del Holoceno tardío), con claras evidencias de procesamiento antrópico, motivó desarrollar un marco de referencia adecuado para la interpretación de conjuntos zooarqueológicos donde esta especie aparece representada.

En términos generales, la mayoría de los trabajos sobre utilidad económica en mamíferos están dirigidos a las grandes presas y son aplicados a la discusión del procesamiento selectivo y al transporte diferencial de las unidades anatómicas (Binford 1978; Jones y Metcalfe 1988; Mengoni Goñalons 1991, Borrero 1990; Savelle y Friesen 1996; Savelle et al. 1996; Belardi y Gómez Otero 1998; Outram y Rowley-Conwy 1998; De Nigris y Mengoni Goñalons 2004; San Román 2009 entre otros). Sin embargo, en la última década las investigaciones arqueológicas han comenzado a explorar el papel que han tenido los pequeños vertebrados en la subsistencia de los cazadores -recolectores (Pardiñas 1999; Quintana 2001; Quintana et al. 2002; Acosta y Pafundi 2005; Cruz et al. 2007; Fernández et al. 2009). En este sentido recientemente se realizó un estudio del rendimiento económico del cauquén común donde se enfatizó la utilidad de expandir este tipo de trabajos a especies consideradas tradicionalmente de "bajo ranking" que aparecen frecuentemente representadas en el registro arqueológico (Tívoli y Pérez 2009:862). Así, estudios en pequeños vertebrados resultaran en marcos de referencia indispensables al momento de evaluar la relevancia que estas especies tuvieron como recursos en el pasado.

Los estudios de utilidad económica son una herramienta importante para la interpretación de los patrones de trozamiento y transporte en los grandes mamíferos (Binford 1978, 1981; Savelle y Friesen 1996; Lupo 1998), pero también aportan información de la estructura corporal de los medianos y pequeños animales para ayudar a entender la relación que estos tenían con los seres humanos (Tivoli y Pérez 2009).
En base a lo expuesto anteriormente, el presente trabajo tiene como objetivo: a) Presentar los valores obtenidos que permitan conocer la anatomía económica de la mara (Dolichotis patagonum); b) Dar a conocer los recientes hallazgos de restos arqueológicos de este roedor en la costa de Río Negro e c) Interpretar a la luz de su anatomía económica los patrones de procesamiento para el consumo de la mara en el sitio estudiado, y discutir el registro resultante a partir del mismo.

Con el fin de cumplir los objetivos propuestos se calcularon el Índice de utilidad de carne (MUI) en sus versiones estandarizadas y sin estandarizar propuestos por Metcalfe y Jones (1988). Con los resultados obtenidos se evalúa el potencial de esta especie como recurso alimenticio y se interpretan los recientes hallazgos arqueológicos en el contexto de la subsistencia de los grupos cazadores-recolectores de norpatagonia.

\section{Características Ecológicas de la Mara}

La mara (Dolichotis patagonum) es un roedor cursorial de gran tamaño, su longitud total varia entre los $60 \mathrm{~cm}$ y $80 \mathrm{~cm}$, y su peso entre $9 \mathrm{~kg}$ y $16 \mathrm{~kg}$ (Nowak 1991). En la actualidad se reconocen dos subespecies Dolichotis patagonum centricota que se encuentra en la Provincia de Catamarca, este de La Rioja, noroeste de Córdoba y suroeste de Santiago del Estero; la otra subespecie Dolichotis patagonum patagonum ocupa la región central de la Provincia de Buenos Aires, sur de Córdoba, San Luis, Mendoza y el sur de Argentina hasta la provincia de Santa Cruz (Campos et al. 2001).

En Patagonia el período reproductivo es altamente estacional, la mayoría de las pariciones ocurre entre mediados de agosto y diciembre (Baldi 2007), donde grupos entre uno y 29 parejas utilizan la misma madriguera para dejar sus crías. Las hembras pueden tener hasta tres crías por camada, pero por lo general solo tienen una sola. La organización social de las maras tiene una combinación única de monogamia y cría comunitaria. Las parejas se acercan al lugar donde dejaron las crías para amamantarlas, mientras las otras parejas forman un circulo alrededor para protegerlas de los predadores (Campos et al. 2001). Preferentemente ubican sus madrigueras en los pastizales abiertos y estepas arbustivas con escasa cobertura vegetal. Mientras que su alimentación se 
centra en vegetación verde y frutos, principalmente gramíneas, (Chloris sp., Pappophorum sp. y Trichloris sp.) y arbustos (Atriplex lampa, Lycium sp. y Prosopis sp.) (Campos et al. 2001).

Si bien su distribución era mucho mas amplia hacia fines del Siglo XIX -se la registró en el partido de Lincoln (Crawford 1884: en Politis et al. 1983)-, en la actualidad se ha visto fuertemente reducida debido a la alteración de su hábitat y a la caza (Díaz y Ojeda 2000).

En vistas a las características ecológicas y etológicas mencionadas anteriormente, como son una marcada elección de hábitat, una reproducción netamente estacional y una gran concentración de individuos en las proximidades de las madrigueras, hacen de esta especie un recurso importante a tener en cuenta por los zooarqueólogos que buscan comprender la subsistencia de los grupos de cazadores-recolectores que habitaron y transitaron el extremo sur de América.

\section{Información etnográfica y antecedentes arqueológicos}

Sobre la base de datos aportados por los exploradores y cronistas del siglo XIX se sabe que la mara fue un recurso utilizado por los grupos cazadores-recolectores de Patagonia durante el período post contacto. Por ejemplo, el naturalista D’Orbigny (1999), que visitó el norte de Patagonia en los años 1828 y 1829, hace referencia que los grupos Patagones vendían a los colonos de Carmen de Patagones alfombras confeccionadas en cueros de diferentes animales, entre ellos la mara. Por su parte Claraz (1988), que realizó el trayecto desde Carmen de Patagones hasta el Río Chubut entre 1865-1866, menciona que el cuero de estos roedores era utilizado para fabricar bolsas para contener agua, mientras que Musters (2005), que viajó con un grupo de Tehuelches desde orillas del río Santa Cruz hasta Río Negro en 1869 , se refiere a las técnicas que empleaban para la caza de las maras, a la utilización de sus pieles para la confección de mantas y al consumo de su carne asada. Por último, Hudson (2007) que visitó las costas del Río Negro en 1871, menciona el hallazgo de antiguos asentamientos aborígenes donde observó gran cantidad de restos óseos de varias especies de animales, entre ellos "dolichotis o liebre patagónica", que habían sido utilizadas como alimentos.
Si bien esta especie ha sido registrada en varios sitios arqueológicos de Pampa, Patagonia y la región Chaco-santiagueña con cronologías que van desde ca 10.000 años AP en cueva Tixi (Quintana 2001; Quintana et al. 2002) hasta ca 440 años AP en el partido de Lobería (Tonni 1985), su representación en la mayoría de ellos está dada por unos pocos elementos óseos (Tabla 1). Por otra parte son muy escasos los sitios en donde se registraron claras evidencias de procesamiento antrópico de este animal. Concretamente en cuatro sitios se recuperaron elementos con huellas de corte, a saber: Campo Laborde datado en ca 8.000 años AP, donde se registró un solo elemento con huellas de corte (Politis y Messineo 2008); Las Olas 11 datado en ca 2.900 años AP donde los autores hacen referencia a huellas de corte sobre huesos de mara, sin especificar en que elemento (Aldazabal et al. 2010); Villa La Punta, un sitio agro-alfarero temprano de la región Chaco-santiagueña datado en ca 1.500 años AP donde se registraron las dos especies (Dolichotis patagonum y D. salinicola) de las cuales solo la especie patagónica evidenció huellas de corte en fragmentos de fémur (del Papa et al. 2010) y en Bajo de la Quinta, Sector 1 Sondeo 2 (los datos de éste último sitio serán discutidos más abajo como caso de estudio).

\section{METODOLOGÍA}

El ejemplar utilizado en el presente trabajo es una hembra adulta que pertenecía al Zoológico Municipal La Máxima de la ciudad de Olavarria (Buenos Aires, Argentina) y que fue hallada muerta en su recinto. El cuerpo del animal fue sometido a una necropsia por parte del veterinario del zoológico Dr. Horacio Grand donde se les retiraron las vísceras y se determinó que murió por causas naturales (Grand com. pers.). El resto del cuerpo se guardó en una heladera hasta el día siguiente cuando se realizó el estudio.

1) Para el cálculo del índice de utilidad económica se siguió el procedimiento propuesto por Mengoni Goñalons (1991) para llama, modificando el número y la distribución de las unidades primarias de trozamiento, por tratarse de un animal significativamente mas pequeño y por lo tanto que podría ser transportado completo al sitio de consumo. En este sentido se tuvieron en cuenta los patrones de 
Tabla 1. Sitios arqueológicos con presencia de D. patagonum. *No se cuenta con una cronología precisa de los restos, sin embargo, la autora hace referencia que para el Holoceno tardío esta especie ya no se registra (Salemme En prensa).

\begin{tabular}{|c|c|c|c|}
\hline Localidad & Datos publicados & Fechado & Referencias Bibliográfica \\
\hline Cueva Tixi & NISP: 4 & $10.045 \pm 95 \mathrm{AP}$ & Quintana 2001; Quintana et al. 2002 \\
\hline Arroyo Seco 2 & NISP: 2 & Holoceno temprano-Holoceno medio* & Salemme En prensa \\
\hline Campo Laborde & $\begin{array}{l}\text { NISP: } 5 \\
\text { NMI: } 1\end{array}$ & ca 8.000 AP & Politis y Messineo 2008 \\
\hline Zanjón Seco 3 & $\begin{array}{l}\text { NISP: } 2 \\
\text { NMI: } 1\end{array}$ & - & Politis 1984; Politis y Salemme 1990 \\
\hline La Toma & $\begin{array}{l}\text { NISP:3 } \\
\text { NMI: } 2\end{array}$ & $995 \pm 65$ AP & Salemme 1987; Politis y Salemme 1990 \\
\hline Cañada de Rocha & Presencia & - & Salemme 1983; 1987 \\
\hline Lobería 1 & $\begin{array}{l}\text { NISP: } 1 \\
\text { NMI: } 1\end{array}$ & $440 \pm 60 \mathrm{AP}$ & Tonni 1985 \\
\hline Fortín Necochea & NISP:4 & $3.630 \pm 60 \mathrm{AP}$ & Pardiñas 1991; Crivelli Montero et al. 1994 \\
\hline El Piche 1 & NISP: 1 & $1.500 \pm 40 \mathrm{AP}$ & Eugenio y Aldazabal 2004 \\
\hline Villa La Punta & $\begin{array}{l}\text { NISP.97 } \\
\text { NMI: } 4\end{array}$ & ca $1.500 \mathrm{AP}$ & del Papa et al. 2010 \\
\hline Nutria Mansa & NME: 7 & 2.700-3.100 AP & Bonomo et al. 2009 \\
\hline Don Aldo 1 & MNE: 2 & ca. 800 AP & Prates et al. 2006 \\
\hline Las Olas 11 & $\begin{array}{c}\text { NISP: } 5 \\
\text { NMI: } 2\end{array}$ & $2.890 \pm 60 \mathrm{AP}$ & Aldazabal et al. 2010 \\
\hline Negro Muerto & NME: 2 & $483 \pm 46$ AP & Prates 2008 \\
\hline Angostura 1 & NME: 1 & $938 \pm 45$ AP & Prates 2008 \\
\hline Playa Alsina o Asina & Presencia & - & Arrigoni et al. 2008 \\
\hline
\end{tabular}

trozamiento empleado por los Nukak para los vertebrados pequeños (menores de $10 \mathrm{~kg}$ ) y medianos (entre 10 y 50kg) (Politis y Martínez 1996). Para la obtención de todas las medidas necesarias se siguieron los siguientes Peso vivo del animal (completo).

2) Se cuerea el animal, se eviscera y se pesan todos los órganos del tórax y abdomen, como también el cuero, sangre, lengua, seso y vísceras (llenas y vacías).

3) Se pesa la carcasa eviscerada (incluyendo cabeza y autopodios).

4) Se divide la carcasa en unidades primarias de trozamiento y se pesan cada una de estas unidades.

a) Cabeza: cráneo y mandíbula.

b) Región torácica: vértebras cervicales y torácicas, costillas y esternebras.

c) Región lumbar: vértebras lumbares.

d) Región pélvica: pelvis, sacro y vértebras caudales.

c) Cuartos delanteros: escápula, húmero, radio, ulna, carpianos, metacarpos y falanges.

d) Cuartos traseros: fémur, tibia, tarcianos, metatarsos y falanges.

5) Se descarnan todos los huesos de las unidades menores y se pesan la carne, grasa y tendones de cada uno de los huesos que componen los cortes primarios. (La grasa y los tendones se pesaran por separado en los casos que se pueda extraer de la carne y del cuero).

6) Se pesan los huesos descarnados y enteros.

7) Se cortan los huesos que contengan médula con una sierra, se extrae la misma y se la pesa.

8) Se hierven y maceran los huesos para desgrasarlos y una vez secos, se los pesa.

9) Se mide el volumen de la cavidad medular de los huesos largos.

En este caso, no se pesaron las vísceras debido a que fueron descartadas al momento de practicarse la necropsia del animal, el día anterior. Tampoco fue posible separar la grasa del cuerpo debido a que este tipo de animal no genera grandes acumulaciones de tejido adiposo entre la carne y el cuero.

En cada paso se registró el peso en gramos de las diferentes partes anatómicas. Para los pesos mayores a 150grs se utilizó una balanza comercial marca Bianchi con una capacidad de $15 \mathrm{~kg}$ y una precisión de $25 \mathrm{gr}$ y para los pesos menores a $150 \mathrm{gr}$ se empleo una balanza digital de precisión. 
Tabla 2. D. patagonum: peso en gramos de las Unidades primarias de trozamiento.

\begin{tabular}{|l|r|}
\hline Pesos generales & Peso (grs) \\
\hline Vivo & 7.300 \\
\hline Carcasa eviscerada con cuero & 5.950 \\
\hline Cuero & 570 \\
\hline Carcasa eviscerada sin cuero & 5.350 \\
\hline Cabeza & 280 \\
\hline Cuartos delantero & 780 \\
\hline Región torácica & 1.160 \\
\hline Región lumbar & 900 \\
\hline Pelvis, sacro y vert. caudales & 640 \\
\hline Cuartos trasero & 1.210 \\
\hline
\end{tabular}

A partir de los datos expresados en las Tablas 2 y 3 se obtuvieron los Índices de utilidad de carne (MUI) propuestos por Metcalfe y Jones (1988).

Una vez obtenidos los citados índices fueron empleados en la interpretación de los materiales recuperados en el Sondeo 2 de la localidad Bajo de la Quinta, Sector 1.

\section{RESULTADOS}

En primer lugar se presentan los pesos de las unidades primarias de trozamiento, el peso vivo del animal, la carcasa eviscerada con y sin cuero y el peso del cuero (Tabla 2). Seguido por los pesos obtenidos para las diferentes unidades anatómicas en que fue seccionada la carcasa de la mara, dado que no se observaron diferencias significativas $\left(\chi^{2}=2,04\right.$; $p>0,05)$ entre los elementos de las extremidades izquierdas y derechas, en la tabla solo se presentan los pesos correspondientes a los miembros del lado derecho del animal (Tabla 3).
De los datos obtenidos se estableció que para un animal de este tamaño, el peso de la carne aprovechable $(3599,2 \mathrm{gr})$ representa $40,3 \%$ del peso vivo del individuo (7300gr) mientras que solo el 0,5\% de su peso corresponden a materias grasas como los sesos y la médula ósea.

En la tabla 4 se presentan los diferentes índices calculados para la mara. El MUI fue calculado como: MUI = peso total de la unidad anatómica - peso del hueso seco (Metcalfe y Jones 1988).

Sobre la base de los índices calculado se desprende que el esqueleto axial, principalmente las regiones torácicas, lumbares y cintura pélvica, presentan la mayor utilidad cárnica, seguida por el fémur y el escápula + húmero.

\section{APLICACIÓN DEL ÍNDICE DE UTILIDAD EN LA INTERPRETACIÓN DEL REGISTRO ARQUEOLÓGICO}

Las investigaciones arqueológicas desarrolladas en la costa del golfo San Matías (Río Negro), han mostrado hasta el momento la presencia de restos óseos de mara en dos localidades: Bahía Final y Bajo de la Quinta (Marani MS). Los materiales analizados en el presente trabajo provienen de esta última localidad.

La localidad Bajo de la Quinta se encuentra ubicada en un campo de médanos vivos cercano a la línea de costa actual (Fig.1). En las zonas deflacionadas entre las dunas, se observan múltiples concheros donde se han registrado abundantes materiales arqueológicos (Borella et al. 2007). En uno de los concheros se recuperaron, junto con otras

Tabla 3. Base de datos generales. Pesos de las diferentes unidades anatómicas de D. patagonum.

* Solo se registró el peso de médula ósea de la ulna, debido que el radio carece de cavidad medular.

\begin{tabular}{|c|c|c|c|c|}
\hline Porciones anatómicas & UA (Hueso+Carne) (grs) & Carne (grs) & Hueso Seco (grs) & Medula ósea (grs) \\
\hline Cabeza & 280 & 70 & 76,3 & - \\
\hline Escápula & 225 & 200 & 7,8 & - \\
\hline Húmero & 110 & 85 & 14,5 & 1,5 \\
\hline Radio y ulna & 50 & 25 & 15,1 & $0,3^{*}$ \\
\hline Metacarpo y Falanges & 25 & 18,1 & 6,9 & - \\
\hline Región torácica & 1.160 & 700 & 67,2 & - \\
\hline Región lumbar & 900 & 750 & 32,2 & - \\
\hline Pelvis, sacro y vert. caudales & 640 & 470 & 42,9 & - \\
\hline Fémur & 440 & 400 & 22,3 & 2,4 \\
\hline Tibia & 135 & 85 & 24,2 & 2,4 \\
\hline Metatarso y falanges & 65 & 41,4 & 23,6 & - \\
\hline
\end{tabular}


Tabla 4. Índices de Utilidad Económica de la Mara (Dolichotis patagonum).

\begin{tabular}{|l|c|c|}
\hline Porciones anatómicas & MUI & MUI(s) \\
\hline Cabeza: & 203,7 & $18,64 \%$ \\
\hline Escápula: & 217,2 & $19,88 \%$ \\
\hline Húmero: & 95,5 & $8,74 \%$ \\
\hline Radio y ulna & 34,9 & $3,19 \%$ \\
\hline Metacarpo y falanges & 18,1 & $1,66 \%$ \\
\hline Región torácica & 1092,8 & $100,00 \%$ \\
\hline Región lumbar & 867,8 & $79,41 \%$ \\
\hline Pelvis, sacro y vert. caudales & 597,1 & $54,64 \%$ \\
\hline Fémur & 417,7 & $38,22 \%$ \\
\hline Tibia & 110,8 & $10,14 \%$ \\
\hline Metatarso y falanges & 41,4 & $3,79 \%$ \\
\hline
\end{tabular}

especies, gran cantidad de restos óseos asignados a la mara, algunos de los cuales presentaron claras huellas de procesamiento antrópico.

Este conchero, identificado como BQ-1-S2, corresponde a un montículo compuesto principalmente de valvas de Mytilus sp. y Aulacomya sp., datado en 3.110 \pm 100 años AP (LP 2238)-rango calibrado 2.919-3.217 años $\mathrm{AP}^{2-}$, que presentaba en su superficie gran cantidad de restos óseos y desechos líticos. Debido a estas características se realizó un sondeo de 1,5 x 0,5 m a efectos de recuperar los materiales en estratigrafía. Por su parte se

2 Las edades fueron calibradas con el Programa CALIB 6.0 a un sigma e incluyen corrección por efecto reservorio calculado localmente en $266 \pm 51$ años (Favier Dubois 2009).
Tabla 5. Lista de especies recuperadas en el sondeo BQ-S1-2.

\begin{tabular}{|l|c|c|}
\hline Taxa & NISP & $\begin{array}{c}\text { Huellas } \\
\text { Culturales }\end{array}$ \\
\hline Aves indet. & 27 & ausencia \\
\hline Eudromia elegans Óseo & 45 & presencia \\
\hline Eudromia elegans Huevo & 43 & ausencia \\
\hline Rhea americana & 9 & presencia \\
\hline Rheidae & 205 & ausencia \\
\hline Mammalia Indet & 184 & presencia \\
\hline Ungulado Indet & 10 & presencia \\
\hline Lama guanicoe & 314 & presencia \\
\hline Roedor Indet & 39 & ausencia \\
\hline Dolichotis patagonum & $\mathbf{8 3}$ & presencia \\
\hline Microcavia australis & 44 & ausencia \\
\hline Ctenomys sp. & 9 & ausencia \\
\hline Dasipodidae Indet & 15 & presencia \\
\hline Chaetophractus villosus & 27 & ausencia \\
\hline Zaedyus pichiy & 16 & ausencia \\
\hline Felidae & 8 & ausencia \\
\hline Otáridos & 19 & ausencia \\
\hline Tortuga & 3 & ausencia \\
\hline indet.. & 8 & ausencia \\
\hline Peces & 1193 & ausencia \\
\hline
\end{tabular}

realizaron recolecciones de superficie del talud oeste y sur del montículo con el objetivo de incluir en el estudio los restos óseos dispersos del conchero, que estaba siendo erosionado por la acción del viento.

En el conjunto arqueofaunístico de este locus se recuperaron 2307 restos óseos de los cuales se identificaron 2114 especímenes correspondientes

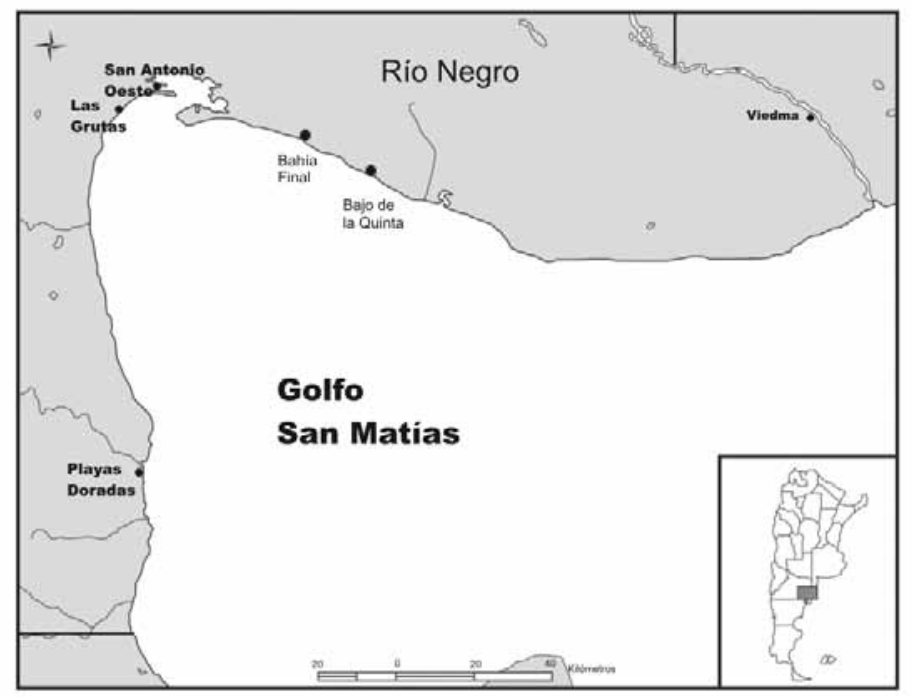

Fig.1 Mapa con las localidades con registro de D. patagonum. 
a más de 16 taxa diferentes, algunos de los cuales evidenciaban huellas antrópicas (Tabla 5). Entre estos últimos se encuentran los restos de D. patagonum. Esta especie esta representada por 83 especimenes óseos. Si bien algunos huesos hallados durante la excavación se encontraban en posición articular (Fig. 2. A y B), se calculó -a partir de los fragmentos proximales de fémur derecho- la presencia de al menos cuatro individuos.

La representación de partes anatómicas de esta especie en BQ1 sondeo 2 se caracteriza por presentar casi la totalidad de los elementos que conforman el esqueleto (a excepción del radio y la fíbula), con un marcado predominio de los elementos de las extremidades (fémur, tibia, escápula, húmero, ulna y metatarsos) y la cabeza (representado por las bulas timpánicas, premaxilares y maxilares) (Tabla 6). Con el objetivo de evaluar si esta representación de partes esqueletarias es el resultado de procesos atricionales, se correlacionó el MAU\% y los valores promedios de densidad mineral ósea de Lepóridos (Pavao y Stahl 1999). Los resultado obtenidos nos muestran que estas variables presentan una correlación moderada y significativa $\left(r_{s}=0,67 ; p<0,05\right)$, lo que indica que dicha representación de partes anatómicas se vió afectada por procesos que favorecieron la preservación de aquellos elementos que presentan mayor densidad mineral ósea. Mientras que la presencia de casi la totalidad de elementos esqueletales nos indica que las carcasas de estos roedores habrían ingresado completas, como es lo esperable para el caso de presas chicas (Tivoli y Pérez 2009).
Tabla 6. D. patagonum Representación de partes esqueletaría en BQ-S1-2.

\begin{tabular}{|l|c|c|c|c|}
\hline Elementos & NISP & NME & MAU & MAU\% \\
\hline Cabeza & 7 & 2 & 2 & $100 \%$ \\
\hline Mandíbula & 2 & 2 & 2 & $100 \%$ \\
\hline Vértebras cervicales & 2 & 2 & 0,28 & $14,28 \%$ \\
\hline Vértebras torácicas & 11 & 11 & 0,91 & $45,83 \%$ \\
\hline Vértebras lumbares & 2 & 1 & 0,14 & $7,14 \%$ \\
\hline Costillas & 16 & 12 & 0,5 & $25 \%$ \\
\hline Escápula & 2 & 2 & 1 & $50 \%$ \\
\hline Húmero & 4 & 3 & 1,5 & $75 \%$ \\
\hline Radio & 0 & 0 & 0 & $0 \%$ \\
\hline Ulna & 3 & 3 & 1,5 & $75 \%$ \\
\hline Metacarpos & 2 & 2 & 0,25 & $12,5 \%$ \\
\hline Sacro & 0 & 0 & 0 & $0 \%$ \\
\hline Pelvis & 2 & 1 & 1 & $50 \%$ \\
\hline Fémur & 9 & 4 & 2 & $100 \%$ \\
\hline Rótula & 1 & 1 & 0,5 & $25 \%$ \\
\hline Tibia & 3 & 2 & 1 & $50 \%$ \\
\hline Fíbula & 0 & 0 & 0 & $0 \%$ \\
\hline Calcáneo & 1 & 1 & 0,5 & $25 \%$ \\
\hline Metatarsos & 8 & 8 & 1,33 & $66,66 \%$ \\
\hline Tarsianos & 4 & 4 & 0,14 & $14,28 \%$ \\
\hline 1Falanges & 2 & 2 & 0,14 & $7,14 \%$ \\
\hline 2 Falanges & 1 & 1 & 0,07 & $3,57 \%$ \\
\hline
\end{tabular}

Por otra parte se registraron in situ dos conjuntos de vértebras torácicas articuladas (Fig.2.A) (porción anatómica de alto rinde económico), evidenciando que esta porción del la columna vertebral "habría" sido consumida y posiblemente descartada como una unidad. Mientras que, la mayor cantidad de huellas del procesamiento antrópico se localizaron en el fémur (unidad anatómica de mayor rinde

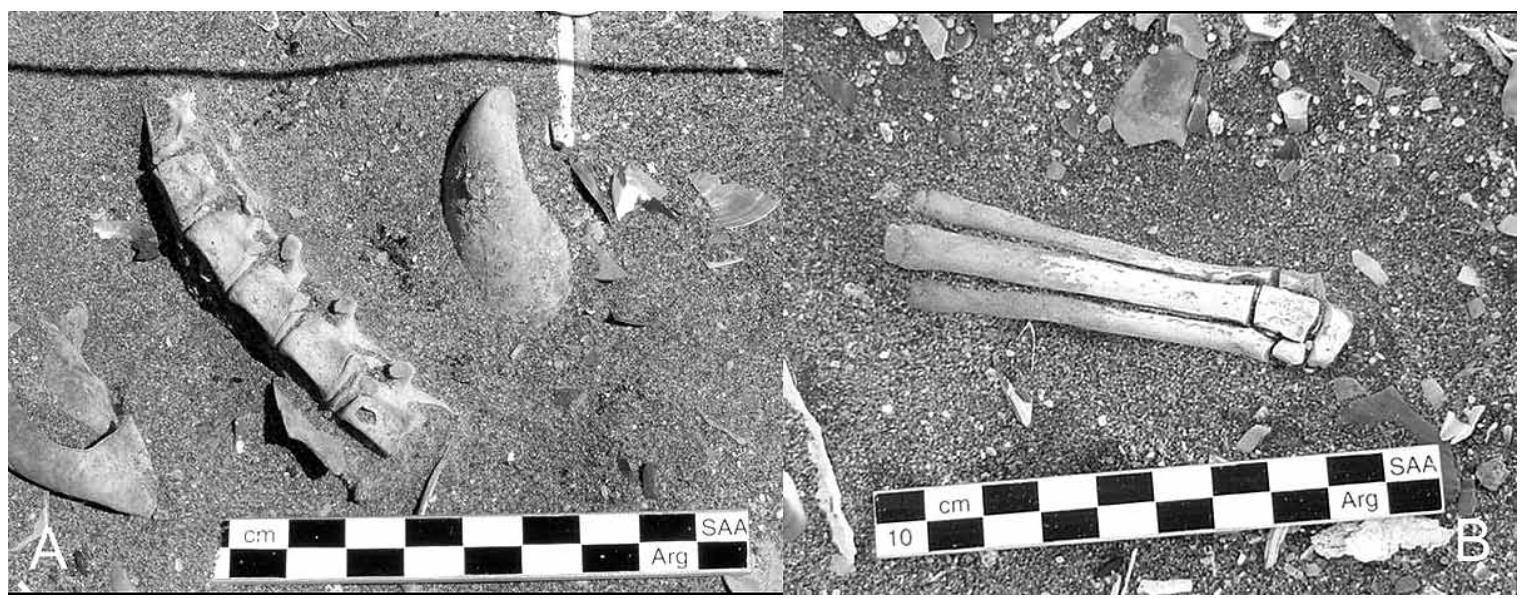

Fig. 2. A) Fragmento de columna vertebral articulada y B) Tarsos y metatarsos articulados de D. patagonum. 


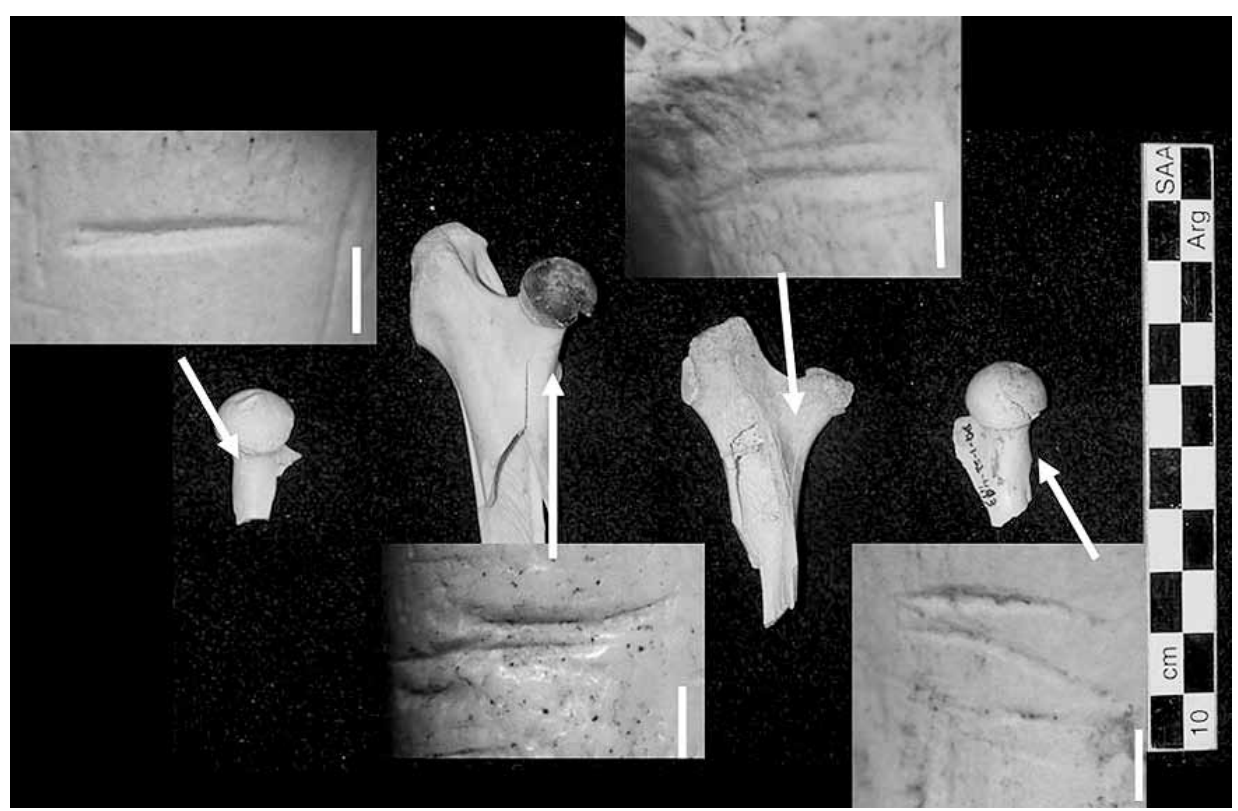

Fig.3. Fémur de D. patagonum con huellas de corte. Imágenes de las huellas de corte tomadas con lupa binocular con un aumento de 20X (Escala 1mm).De izquierda a derecha (especímenes BQ-1-S2-543; BQ-1-S2-472; BQ-1-S2-471 y BQ-1-S2-483).

después del esqueleto axial) (Fig. 3). Estas evidencias de procesamiento, como huellas de corte, alteración térmica y lascados de percusión, registradas en el fémur nos aporta información de cómo habría sido el procesamiento de este animal. De esta forma, a partir de la localización de las huellas de corte en la base del cuello de la articulación proximal de los especimenes del fémur (Fig. 3), pueden ser inferidas como evidencia de desarticulación de los cuartos traseros (Binford 1981). Por otra parte, uno de los especímenes (BQ-1-S2-472) presenta alteración térmica localizada en el cabeza articular quedando el resto del hueso sin quemar (Fig.4.A). Otros autores han correlacionado esta evidencia con un modelo de cocción, donde la temperatura ocasiona la retracción de la carne, dejando expuesto al fuego una porción del hueso (Pardiñas 1999:285; Santiago 2004:6). Por último, este mismo espécimen, presenta fractura helicoidal en la zona de la diáfisis, con dos muescas de percusión en forma de escotadura semi lunar (Fig.4.B). Estas son características diagnósticas de fractura intencional del hueso en estado fresco que

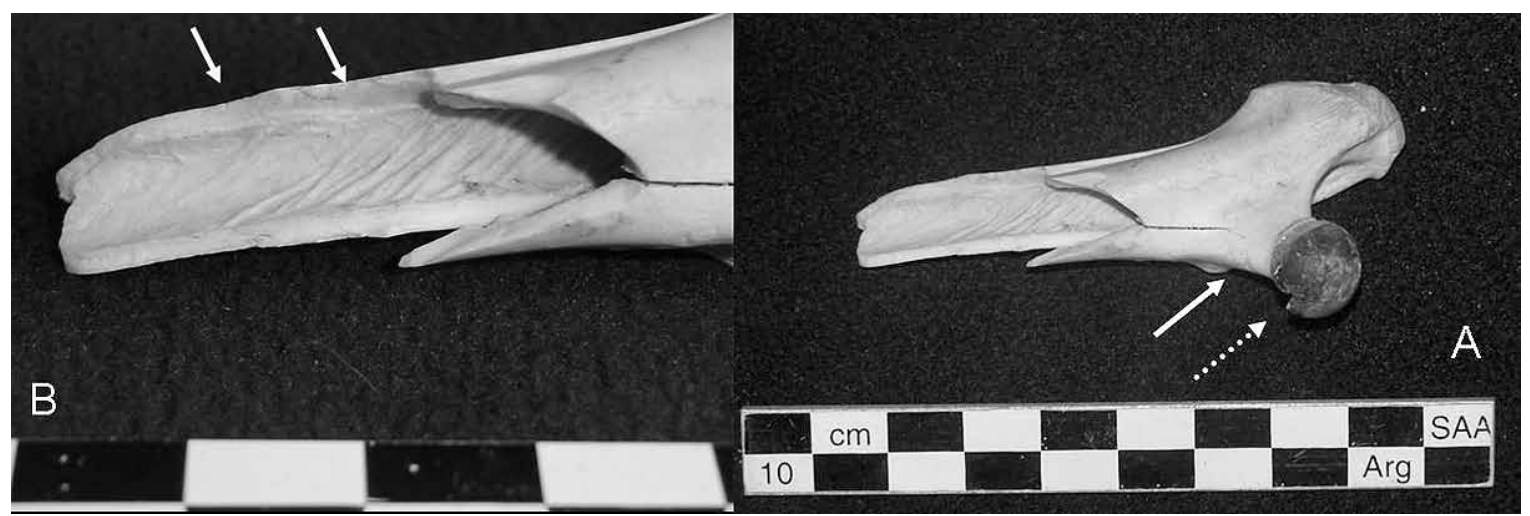

Fig. 4. Fémur de D. patagonum (Espécimen BQ-1-S2-472). A) La flecha en línea discontinua indica la alteración térmica, la flecha continua indica la localización de las huellas de corte. B) Las flechas indican las muescas de percusión. 
varios autores han interpretado como evidencia de aprovechamiento de médula ósea (Binford 1981) u obtención de formas base para la confección de instrumentos (Gifford-González 1989; Mengoni Goñalons 1999; Miotti 1998). En este caso se interpreta como consecuencia del consumo de medula ósea al estar asociadas a otras evidencias de consumo.

En lo que respecta al conjunto arqueofaunístico (a excepción de los peces) se observaron una muy baja incidencia de marcas de carnívoros, roedores o pisoteo ( $1,26 \%$; $0,09 \%$ y $0,45 \%$ respectivamente), mientras que las marcas de raíces y de acción química producida por las mismas presentaron valores un poco más elevados (17,9\% y 15\% respectivamente). Por otro lado, en relación a las huellas de actividad antrópica se registraron huellas de corte en un 2,9\% del total de los especímenes, mientras que las huellas de percusión se observaron en el 3,1\% Los porcentajes antes mencionados estarían indicando que los agentes naturales, tales como la acción de carnívoros y roedores, no habrían afectado significativamente la integridad de la muestra arqueofaunística, mientras que las marcas de raíces estarían indicando la estabilización de los sedimentos que cubría el conchero a partir de la formación de un suelo.

Sobre la base de las variables tafonómicas analizadas, y las evidencias culturales que presentan ciertos especímenes de $D$. patagonum podemos sostener que esta especie habría ingresado al sitio como consecuencia del descarte por consumo humano.

\section{DISCUSIÓN Y CONCLUSIONES}

Si bien el ejemplar de mara utilizado para calcular este índice corresponde a un animal criado en cautiverio, y es bien sabido que esta especie en estado silvestre alcanza un mayor desarrollo (Nowak 1991), los pesos obtenidos y los índices no estandarizados podrían considerarse subestimados. Sin embargo, dado que las características anatómicas y las proporciones corporales de animales en cautiverio y en estado silvestre son iguales, los índices estandarizados no se ven afectados. Por lo tanto la información generada puede ser utilizada como base en las interpretaciones de las muestras arqueofaunísticos donde se registre esta especie. Por otra parte, la escasa cantidad de médula ósea registrada en los huesos largos (menos del 0,15\% del peso total) hace suponer que la obtención de esta no afectaría en forma significativa la representación de partes esqueletarias en un sitios arqueológico.

Como fue reconocido por Binford (1978) y Lyman et al. (1992), entre otros, los índices de utilidad económica nos proveen uno de varios marcos de referencia, con los cuales se pueden hacer inferencias sobre la representación de partes esqueletarias dentro de un sitios arqueológico (Savelle y Friesen 1996). La aplicación del índice de utilidad económico de mara, permitió determinar que las partes de mayor rinde cárnico, como ser la porción torácica de la columna vertebral y el fémur, se encontraron descartadas en el conchero. Asimismo, las porciones de mayor valor económico como el fémur presentaron claras evidencias que refieren a práctica de cocción para el consumo de carne (Pardiñas 1999). Por último, las huellas culturales registradas en elementos de los cuartos traseros permiten inferir que los mismos fueron desarticulados de la pelvis, expuestos a una fuente de calor (posiblemente asados) y luego, una vez extraída la carne del hueso, habrían sido fracturados intencionalmente para el consumo de su médula.

Lo expuesto en el párrafo anterior podría ser considerado como un patrón preliminar sobre el procesamiento y consumo de la mara por los grupos cazadores-recolectores, la cual deberá ser necesariamente cotejada a futuro con nuevas evidencias que permitan discutir lo aquí presentado.

Por último, la obtención de los índices de utilidad económica para esta especie de roedor ayudará a comprender como la anatomía de este vertebrado mediano influyó en el patrón de su procesamiento y el papel que ocupó este recurso en la subsitencia de los grupos humanos que habitaron la costa del golfo San Matías, como también de otras áreas dónde estuvo disponible esta especie en el pasado.

\section{AGRADECIMIENTOS}

A los Dres. Florencia Borella y Cristian Favier Dubois por su constante apoyo, aporte de ideas y discusiones que enriquecieron este trabajo. A la Agencia de Cultura de Río Negro y al CODEMA por los permisos otorgados para el desarrollo de los trabajos. A la Sra. Irma Stábile y al Sr. Bonifacio (dueña y encargado de la Estancia La Madreselva) por permitirnos realizar nuestro trabajo en su establecimiento. 
Especialmente agradecer a la Dra. Sandra Botasi y al Vet. Horacio Grand por permitirnos el acceso a los restos de la mara en el Parque Zoológico Municipal "La Máxima" de la ciudad de Olavarría para los estudios de utilidad económica. A Agustín Venzí por su valiosa colaboración en el procesamiento de la mara. A Daniel Rafuse por la traducción del Abstract. Este trabajo forma parte de mi plan de beca tipo II Conicet, en el marco de los estudios que se vienen desarrollando en la costa rionegrina, financiados por Agencia (PICT 38264) y CONICET (PIP 6415 y 112-200801-00756). Finalmente quiero agradecer a los evaluadores anónimos por sus comentarios y sugerencias.

\section{BIBLIOGRAFÍA}

ACOSTA, A. y L. PAFUNDI 2005. Zooarqueología y tafonomía de Cavia aperea en el humedal del Paraná inferior. Intersecciones en Antropología, 6: 59-74.

ALDAZABAL, V., E. EUGENIO y M. SILVERA 2010. Arqueología del sector costero al sur de Bahía de San Blas: Sitio las Olas 11. Arqueología Argentina en el Bicentenario de la Revolución de Mayo. Bárcena, J.R. y H. Chiavazza (Editores.) Libro de actas del XVII Congreso Nacional de Arqueología Argentina. Mendoza. Tomo I: 303-308.

ARRIGONI, G., M. ANDRIEU y C. BAÑADOS 2006. Arqueología de Cazadores Recolectores prehistóricos en la costa central del golfo San Jorge. En Arqueología de la Costa Patagónica, perspectivas para la conservación, editado por I. Cruz y S. Caracotche, Capítulo 6, pp. 91-107. UNPA, Río Gallegos.

BALDI, R. 2007. Breeding success of the endemic mara Dolichotis patagonum in relation to habitat selection: Conservation implications. Journal of Arid Environments, 68: 9-19.

BELARDI, J.B. y J. GÓMEZ OTERO 1998. Anatomía económica del Huemul (Hippocamelus bisulcus): una contribución a la interpretación de las evidencias arqueológicas de su aprovechamiento en Patagonia. Anales del Instituto de la Patagonia, Ser. Cs. Hs., 16:195-207.

BINFORD, L.R. 1978. Nunamiut Ethnoarchaeology. New York: Academic Press.

1981. Bones. Ancient Men and Modern Myths. New York: Academic Press.

BONOMO, M., A. F. ZUCOL, B. GUTIÉRREZ TÉLLEZ, A. CORADEGHINI y M. S. VIGNA 2009. Late Holocene palaeoenvironments of the Nutria Mansa 1 archaeological site, Argentina. Journal of Paleolimnology, 41:273-296
BORELLA, F., C. MARIANO y C. FAVIER DUBOIS 2007. Procesos tafonómicos en restos humanos de superficie en la localidad arqueológica de Bajo de la Quinta, golfo San Matías (Río Negro) Argentina. En: Arqueología de Fuego-Patagonia. Levantando piedras, desenterrando huesos...y develando arcanos. Morello, F., M. Martinic, A. Prieto y G. Bahamonde Eds. Ediciones CEQUA, Punta Arenas. Pp.403-410.

BORRERO, L. A. 1990. Fuego - Patagonia bone assemblages and the problem of comunal guanaco hunting. En Hunters of the recent past, editado por L. B. Davis y B. O. K. Reeves, pp. 373-406. Unwin Hyman, Londres. CAMPOS, C. M., M.F. TOGNELLI y R. A. OJEDA 2001. Dolichotis patagonum. Mammalian species 632:1-5.

CLARAZ, J. 1988. Diario de Viaje de Exploración al Chubut. 1865-1866. Marymar, Buenos Aires.

CRIVELLI MONTERO, E., E. EUGENIO, U. PARDIÑAS y M. SILVEIRA 1994. Archaeological Investigation in the plains of the Provinces of Buenos Aires, Llanura Interserrana Bonaerense. Quaternary of South America and Antartic Peninsula, 10:167-207.

CRUZ, I, S. MUÑOZ y A. F. ZANGRANDO 2007. La interpretación de los restos de animales pequeños en la arqueología patagónica: Estado de la cuestión y perspectivas. En Arqueologia de Fuego-Patagonia. Levantando piedras, desenterrando huesos...y develando arcanos. Editado por: Morello, F, M. Martinic, A. Prieto y G. Bahamonde. Ediciones CEQUA, Punta Arenas. Pp. 15-22.

DE NIGRIS, M. E. y G. L. MENGONI GOÑALONS 2004. The guanaco as a source of meat and fat in the Southern Andes. En The zooarchaeology of fats, oils and dairying, editado por J. Mulville y A. Outram, pp. 160-166. Oxbow Books, Oxford.

DEL PAPA, L.; L. DE SANTIS y J. TOGO 2010. Consumo de roedores en el sitio Villa la Punta, agro-alfarero temprano de la región Chaco-Santiagueña. Intersecciones en Antropología, 11:29-40

DIAZ, G B. y R A. OJEDA (eds). 2000. Libro Rojo de los mamíferos Amenazados de la Argentina. 2000. Sociedad Argentina para el Estudio de los Mamíferos, SAREM. $106 \mathrm{pp}$

D’ORBIGNY, A. 1999. Viaje por América meridional. Tomo 2. Emecé, Buenos Aires

EUGENIO, E. y V. ALDAZABAL 2004 Los cazadoresrecolectores del litoral marítimo del área de Bahía San Blas, Provincia de Buenos Aires. En Contra viento y marea. Arqueología de Patagonia. M. T. Civalero, P. M. Fernández y A. G. Guraieb (Eds.), pp: 687-700 INALP-SAA. Buenos Aires 
FAVIER DUBOIS, C.M. 2009. Valores de efecto reservorio marino para los últimos 5.000 años obtenidos en concheros de la costa atlántica norpatagónica (golfo San Matías, Argentina). Magallania, 37(2): 139-147

FAVIER DUBOIS, C. M. y F. BORELLA 2007 Consideraciones acerca de los procesos de formación de concheros de la costa norte del golfo San Matías, Río Negro. Cazadores Recolectores del Cono Sur. Revista de Arqueología, 2: $151-165$

FAVIER DUBOIS, C. M., F. BORELLA; L. M. MANZI, M. CARDILLO, S. LANZELOTTI, F. SCARTASCINI, C. MARIANO y E. BORGES VAZ 2006 Aproximación regional al registro arqueológico de la costa rionegrina. En Arqueología de la costa Patagónica. Perspectivas para la Conservación, editado por I. Cruz y M. S. Caracotche, pp. 50-68. Universidad Nacional de la Patagonia Austral, Río Gallegos.

FERNÁNDEZ, F.J.; MOREIRA, G.J.; BALLEJO, F. \& DE SANTIS, L.J.M. 2009. Novedosos registros de aves exhumadas del sitio arqueológico "Laguna El Sosneado (LS-3)" para el Holoceno tardío en el sur de Mendoza: aspectos tafonómicos. Intersecciones en Antropología, 10: 327-342

FIORE, D y F. BORELLA. 2010. Geometrías delicadas. Diseños grabados en cáscaras de huevo de Rheidae recuperados en la costa norte del golfo San Matías, Río Negro. Intersecciones en Antropología, 11: 277-293

GIFFORD-GONZÁLEZ, D. 1989 Ethnographic analogues for interpreting modified bones: some cases from East Africa. En Bone Modification, editado por Robson Bonnichsen y Marcella Sorg, pp. 179-246. Center for the Study of the First Americans, Orono.

HUDSON, W. H 2007 [1893]. Días de ocio en la Patagonia. Diario de un naturalista (1893). Ediciones Continente. Buenos Aires

JONES, K. T. y D. METCALFE 1988 Bare bones archaeology: Bone marrow indices and efficiency. Journal of Archaeological Science, 15: 415-423

LUPO, K. 1998 Experimentally derived extraction rates for marrow: Implications for body parts exploitation strategies of Plio-Pleistocene hominid scavengers. Journal of Archaeological Science, 25: 657-75

LYMAN, R. L., SAVELLE, J. M. y P. WHITRIDGE 1992. Derivation and application of a meat utility index for phocid seals. Journal of Archaeological Science, 19:531-555.

METCALFE, D. y K. J. JONE 1988. A reconsideration of animal body-part utility indices. American Antiquity, 53(3):486-504.
MENGONI GOÑALOS, G. 1991. La llama y sus productos primarios. Arqueología. Revista de la Sección Prehistoria, 1:179-196.

1999. Cazadores de Guanacos de la Estepa Patagónica. Sociedad Argentina de Antropología, Buenos Aires.

MIOTTI, L. 1998. Zooarqueología de la meseta central y costa de Santa Cruz. Un enfoque de las estrategias adaptativas aborígenes y los paleoambientes. Museo Municipal de Historia Natural, Mendoza.

MUSTERS, G. 2005 [1871]. Vida entre los patagones. El Elefante Blanco. Buenos Aires.

NOWAK, R. M. 1991. Warlker's Mammals of the World Fifth Edition Volume II. The Johns Hopkins University Press. Baltimore and London, pp. 913-914.

OUTRAM, A. y P. ROWLEY-CONWY 1998. Meat and Marrow Utility Indices for Horse (Equus). Journal of Archaeological Science 25: 839-849.

PARDIÑAS, U. 1991. Roedores, marsupiales y edentados en la secuencia e de Fortín Necochea, General La Madrid, Buenos Aires, implicancias paeloambientales. Boletin del Centro, 2:139-153

1999 Tafonomía de microvertebrados en yacimientos arqueológicos de Patagonia (Argentina). Arqueología, 9: 265-340.

POLITIS, G. 1984. Arqueología del Área Interserrana Bonaerense. Tesis doctoral no publicada. Facultad de Ciencias Naturales y Museo, Universidad Nacional de La Plata. MS

POLITIS, G y G. MARTÍNEZ 1996. La cacería, el procesamiento de las presas y los tabúes alimenticios. Nukak. Politis, G. Cap.5: 231-279.

POLITIS, G. y P. MESSINEO 2008. The Campo Laborde site: New evidence for the Holocene survival of Pleistocene megafauna in the Argentine Pampas. Quaternary International 191: 98-114

POLITIS, G. y M. SALEMME 1990. Pre-Hispanic mammal exploitation and hunting strategies in the eastern Pampa subregion of Argentina. En: Hunters of the Recent Past. Davis, L. y B. Reeves (Eds). Unwin Hyman. London. Pp:352-372.

POLITIS, G., P. TONNI y F. FIDALGO 1983. Cambios corológicos de algunos mamíferos en el área interserrana de la Provincia de Buenos Aires Durante el Holoceno. Ameghiniana, XX (1-2): 72-80.

PRATES, L. 2007. Arqueología del Valle Medio del Río Negro (Provincia de Río Negro). Tesis doctoral no publicada. Facultad de ciencias Naturales y Museo, Universidad Nacional de La Plata. MS.

PRATES, L., G. MARTINEZ y C. SCABUZZO 2006. Evidencia arqueológica del Holoceno Tardío Final en el curso medio 
del Río Colorado (Provincia de Río Negro): Sitio Don Aldo 1. Cazadores-recolectores del Cono Sur, 1:163-177.

QUINTANA, C. 2001. Composición y cambio en la secuencia faunística. En Cueva Taxi: Cazadores y recolectores de las Sierras de Tandilla Oriental. 1 Geología, Paleontología y Zooarqueología. Eds. D. Mazzanti y C. Quintana. Universidad Nacional de Mar del Plata, La PLata. Cap. 4:37-64.

QUINTANA, C., F. VALVERDE y D. MAZZANTI 2002: Roedores y lagartos como emergentes de la diversificación de la subsistencia durante el Holoceno de las sierras de Tandilia, Argentina. Latin American Antiquity, 13(4): 455-473.

SALEMME, M. 1983. Distribución de algunas especies de mamíferos en el Noreste de la Provincia de Buenos Aires durante el Holoceno. Ameghiniana, 20(1-2): 81-94.

SALEMME, M. 1987. Paleoetnozoologia del sector bonaerense de la región Pampeana, con especial atención a los mamíferos. Tesis doctoral no publicada. Facultad de ciencias Naturales y Museo, Universidad Nacional de La Plata. MS.

SAN ROMÁN, M. 2009. Anatomía económica de Otaria flavescens [Shaw, 1800]. Zooarqueología y tafonomía en el confín del mundo. Editores López M., P, I. Cartagena
F., C. García P. y F. Mena L. Facultad de Estudios del Patrimonio Cultural. Universidad Internacional SEK, Santiago. Pp. 196-179.

SANTIAGO, F. 2004 Los roedores en el "menú" de los habitantes de Cerro Aguará (Provincia de Santa Fé): Su análisis arqueofaunístico. Intersecciones en Antropología, 5: 3-18.

SAVELLE, J.M. y T.M. FRIESEN 1996. An Odontocete (Cetacea) Meat Utility Index. Journal of Archaeological Science, 23: 713-721.

SAVELLE, J.M, T.M. FRIESEN y R. L. LYMAN 1996. Derivation and Application of an Otariid Utility Index. Journal of Archaeological Science 23: 705-712.

TRIVOLI, A. y A. PÉREZ 2009. Rendimiento económico del cauquén común (Chloephaga picta, Familia: Anatidae). En Arqueología de la Patagonia: Una mirada desde el confín del mundo. Compiladores: Santiago, F., M. Salemme, M. Álvarez, E. Piana, M. Vázquez y M. E. Mansur. Editorial Utopías, Ushuaia. Tomo 2: 853-864.

TONNI, E. 1985. Mamíferos del Holoceno del Partido de Lobería, provincia de Buenos Aires. Aspectos paleoambientales y bioestratigráfico del Holoceno del sector oriental de Tandilia y área Interserrana. Ameghiniana, 22(3-4):284-288. 